Reprod. Nutr. Dévelop., 1987, 27 (1 B), 109-128.

\title{
Évolution historique des concepts de faim, satiété et appétits
}

F. GALLOUIN, J. LE MAGNEN (*)

Institut National Agronomique Paris-Grignon, 16, rue Claude-Bernard, 75231 Paris Cedex 05.

(*) Collège de France.

11. place Marcellin-Berthelot, 75005 Paris.

Dès l'Antiquité, les naturalistes se sont intéressés aux notions de faim, satiété et appétits; leurs spéculations avaient pour origine les sensations associées au besoin de manger, expérience quotidienne parmi les plus pressantes de l'homme de la naissance à la mort. La faim, la satiété et les appétits étaient donc généralement perçus comme des états subjectifs propres à l'homme. Ils ne pouvaient pas s'appliquer à l'animal, comme l'homme en quête de nourriture.

Au début du $19^{e}$ siècle, les recherches portèrent essentiellement sur les causes des sensations et les termes de faim-satiété et appétits continuèrent à désigner ces états subjectifs. Au cours des dernières décennies les recherches expérimentales chez l'animal portant sur les déterminants physiologiques nerveux et métaboliques du comportement alimentaire, et de ses relations avec la régulation nutritionnelle de l'organisme, ont conduit à une connaissance avancée des mécanismes physiologiques, communs à l'homme et à l'animal. Dès lors, une étape décisive de l'évolution des concepts était franchie.

L'objet de cette étude est de présenter les principales théories et les expériences princeps qui constituent des étapes historiques. Cette étude s'inscrit dans une série de recherches en nutrition animale et humaine que I'un de nous (Gallouin, 1980 ) a déjà eu l'occasion de présenter dans ce périodique. Dans une première partie nous définirons les termes de " faim ", "satiété " et " appétits " et l'évolution historique de ces définitions. Dans une seconde partie, nous retracerons l'évolution de ces concepts depuis l'époque héllénistique jusqu'au début du 19 e siècle ainsi que des expériences majeures réalisées entre 1756 et 1925.

\section{Approche sémantique.}

Trois modes de définition d'un terme, c'est-à-dire du concept abstrait ou concret qu'il désigne, sont possibles : la définition étymologique, la définition d'usage, enfin la définition normative ou scientifique. La référence à l'étymologie ne peut justifier sa définition. Un nombre considérable de termes sont utilisés dans un sens différent et parfois inverse de celui qu'avait le terme dans la langue d'origine. La définition par l'usage commun du mot est celle qu'enregistrent les dictionnaires ; elle n'est qu'approximative et tel usage particulier, cité à partir de 
la littérature, ne peut lui donner une valeur normative. Pour tous les concepts, les objets et les faits qui relèvent de l'analyse scientifique, une vraie définition émane de la connaissance des phénomènes, de leur description et des causes qui les déterminent. Une telle définition est " normative » et s'impose comme " l'autorité de la chose jugée ". La science, a-t-on dit, est un langage.

\section{- La faim.}

Le mot est issu du latin : "fames " et la plupart des dictionnaires donnent du terme une définition restrictive limitée à la perception sensorielle. Le dictionnaire du C.N.R.S., dit " Trésor de la langue française » (1974), comme le Robert (1965) reprennent, à peu de choses près, la définition du Littré (1876) : « la faim est une sensation que fait éprouver le besoin ou l'envie de manger ». Le Quillet (1968) est plus précis : " La faim est une sensation interne qui réapparaît à intervalle régulier chez l'homme et les animaux ". Un pas décisif est franchi dans l'Encyclopédie des Sciences et des Techniques (1971) : " La faim est une manifestation physiologique d'un instinct fondamental. C'est la sensation qui pousse les êtres vivants à satisfaire leur nécessité en aliment ").

A l'issue d'un nombre considérable de travaux expérimentaux sur l'animal de laboratoire et le sujet humain durant ces quarante dernières années (cf. Le Magnen, 1986), la définition scientifique suivante peut être proposée : « La faim est un état d'éveil spécifique ou de "motivation " du système nerveux central, provoqué par des signaux internes résultant du déficit énergétique de l'organisme requérant l'apport d'aliment et/ou par des stimulations sensorielles externes issues des aliments. Cet état est associé à une perception identifiée chez I'homme, dite "sensation de faim ".

Cet état conduit l'animal, comme l'homme, à rechercher, accepter et ingérer des matériaux qu'il connaît être des aliments. Cette définition issue des travaux contemporains ainsi que celle ci-dessous de "appétit ", est voisine de celle que donnait déjà Claude Bernard en 1848.

\section{- La satiété.}

Selon Robert, le terme est rencontré dans la langue française écrite dès 1120 . Etymologiquement, il provient du latin "Satis " : assez. Le terme est utilisé dans un sens général pour désigner aussi la satisfaction, et par conséquent la disparition d'un besoin par un acte consommatoire. Le mot satisfaction, du latin " satisfactio ", se rapporte initialement à la réparation d'une faute. Le terme s'applique, dans ce sens général, à la faim, à la soif et au rapprochement sexuel. Concernant la faim, tous les dictionnaires, depuis Littré, s'inspirent de cette définition avec les expressions usuelles : manger à satiété, jusqu'à satiété. Une connotation péjorative distingue le "rassasiement.» de la "satiété ». Cette distinction est ignorée des auteurs anglais pour lesquels "satiation " et "satiety " sont synonymes. Cette confusion terminologique a conduit la plupart des auteurs spécialisés de langue anglaise à une confusion de deux concepts : le rassasiement est spécifique des aliments consommés. Rassasié pour un aliment donné, l'homme - et aussi l'animal - peut reprendre la consommation d'un autre aliment. L'addition des rassasiements partiels conduit à une satiété complète. Le sujet se dit alors repu 
(" sated » en anglais). La satiété est l'état de non-faim qu'a introduit un rassasiement partiel ou total. Cet état de "non-stimulation à manger » se prolonge jusqu'à la réapparition de cette stimulation à manger, ou « de faim ». Comme pour la faim, il n'existe aucune raison de limiter l'usage du terme à la sensation perçue par l'homme au moment de l'acquisition et durant la prolongation de l'état de satiété. Le terme est, dans toute les langues, utilisé, et à juste titre, dans l'étude du comportement animal.

- Appétit. - Palatabilité. - Les appétits. - L'appétence.

Ces quatre termes caractérisent des " états de stimulation à manger » de I'homme et des animaux.

Appétit est issu du latin "apetitus" = désir. Ce sens étymologique de " désir " se retrouve dans l'usage et la définition de la plupart des dictionnaires. Par exemple, Robert (1965) définit " appétit » comme " un penchant naturel, un instinct, le désir de quelque chose ». Appliqué aux aliments, le terme est défini de façon fantaisiste par la plupart des dictionnaires, en outre, ces définitions ne différencient pas l'appétit, ou les appétits, de la faim. Pour l'Encyclopédie des Sciences et des Techniques, "l'appétit est le premier degré de la sensation de faim ". Cette notion est erronée : appétit = degré de la faim, se retrouve dans le langage scientifique des auteurs des 18 et $19^{\text {e }}$ siècles. La définition de Littré, " désir de manger " est reprise par le dictionnaire du C.N.R.S. (1974). Cependant Littré soupçonne la définition exacte lorsqu'il écrit: " La faim est essentiellement l'expression d'un besoin. Elle ne peut être ni provoquée, ni excitée comme l'appétit. Celui-ci se prononce pour tel aliment de préférence à tel autre. "

La définition scientifique de l'appétit, est l'attirance et la stimulation à manger que suscitent les aliments en général, ou, à des degrés divers, des aliments particuliers, dans l'état de faim. Des aliments sont, par leurs caractéristiques sensorielles propres, des "stimuli conditionnés" des appétits. Dans cette définition, " appétit » est un quasi synonyme de "palatabilité ». L'appétit pour un (ou des) aliment est le corollaire de la palatabilité de cet (ou de ces) aliment.

La palatabilité est appréciée par la réponse ingestive de l'animal en fonction des qualités sensorielles de l'aliment. On dit que la palatabilité est "faimdépendante " car son influence sur l'ingestion dépend de "l'état de faim " et de son degré. Elle est "faim-spécifique ": les stimuli sensoriels, issus d'un aliment ou d'une solution palatable dans l'état de faim, ne le sont plus dans l'état de soif et réciproquement. Enfin la palatabilité est sensoriellement spécifique, c'est-à-dire spécifique des caractéristiques sensorielles discriminables ou "flaveur \# de chaque aliment concret. Ce terme de "palatabilité ", introuvable dans les dictionnaires actuels, n'est pas un néologisme : il existait dans la langue française. Suivant l'Oxford English Dictionnary, il est - venant du français - introduit dans la langue anglaise en 1669. Ce même dictionnaire en donne une définition imparfaite : "pleasant to the taste, agreable to the mind " (Ed. de 1964, p. 873).

La littérature scientifique distingue des appétits sensoriellement déterminés. Dans ce cas, le terme est synonyme de "consommation ", dont on a observé la variation en fonction de la qualité sensorielle de l'aliment ; l'appétit protéique est 
un appétit spécifique pour la viande. L'appétit calorique est le désir d'ingérer des aliments en fonction de leur valeur énergétique.

Le terme d'appétence est, dans l'usage commun, un synonyme approximatif d'appétit, il ne peut donc être appliqué comme adjectif qualificatif de l'aliment. II n'est pas utilisé dans le langage scientifique.

\section{- Appétibilité. - Ingestibilité.}

En fonction de leurs qualités organoleptiques les aliments seront plus ou moins "préférés ». Cette notion de " préférence » est fondamentale car la quantité ingérée est souvent un facteur limitant des productions animales. Plusieurs termes sont proposés pour rendre compte de cette échelle de valeur. On dit qu'un aliment est plus ou moins palatable, ou préféré par rapport à un aliment de référence : ce niveau de palatabilité peut se mesurer par la vitesse d'ingestion au début du repas.

Les zootechniciens utilisent le terme " d'appétibilité 》 proposé, selon Jarrige (1986) par Rebischung en 1950 comme équivalent à la " palatability 》 des AngloSaxons, appétible est donc un synonyme de palatable. Ce mot existe dans le Littré et le Larousse Encyclopédique de 1960 : " appétibilité : faculté d'appéter ». On peut I'utiliser, selon Jarrige, "pour désigner les qualités organoleptiques des aliments qui suscitent l'appétence. On compare l'appétibilité des aliments en les mettant simultanément à la disposition des animaux " et on admet que les aliments les plus rapidement et/ou les plus consommés sont les plus appétibles pour l'animal.

Selon Jarrige : "Le terme d'ingestibilité est employé pour désigner la quantité d'un fourrage qui est volontairement ingéré par le Ruminant lorsqu'il lui est offert, ad libitum, comme seul aliment. Même si ce néologisme a une "allure qualitative ", nous lui donnons une signification quantitative. On compare l'ingestibilité de différents fourrages en les offrant successivement aux mêmes animaux ou simultanément à des animaux comparables ayant la même capacité d'ingestion. " Elle détermine la taille des repas, souvent par l'intermédiaire des effets post-ingestifs des fourrages qui résultent de leurs caractéristiques physico-chimiques ; l'appétibilité en est une des composantes."

\section{De la période hellénistique au 18 e siècle.}

Les concepts de faim, satiété, appétits, sont présents chez Hippocrate (-400) dans deux ouvrages : "Du Régime " et "Aphorismes " : 10 l'appétit réel (gastrique) est celui qui prédispose l'individu à ingérer ; $2^{\circ}$ l'appétit sensuel (fictif) qui n'a pas de fonction restauratrice, mais flatte les organes des sens; $3^{\circ}$ un appétit d'habitude est lié à l'heure des repas. D'après ces textes Hippocrate distingue la faim de l'appétit et il existe des prises alimentaires "d'habitude ». De plus, l'appétit dépend des besoins de l'organisme; la quantité ingérée augmente lors de la croissance et de l'exercice musculaire (Aph. 2-1). Le rapprochement entre la quantité ingérée et la dépense pour la croissance ou l'activité physique nous semble intéressant à souligner. On ne trouve pas d'indication sur le comportement alimentaire de l'animal. 
L'apport d'Aristote (384-322 av. J.C.) est considérable dans les œuvres traitant de l'histoire naturelle des animaux: "Histoire des animaux ", "Les parties des animaux ", " De l'âme », " De la génération des animaux " (vers 347-342 av. J.C.).

Au livre 1,1 de l'Histoire des animaux, les espèces sont classées selon leur régime alimentaire, certaines sont carnivores, d'autres sont frugivores, d'autres amassent des aliments de réserve... En ce qui concerne les herbivores, le paragraphe 7 traite de la nourriture des Bovins et le paragraphe 8 de celle des Equidés. Aristote insiste sur la relation entre ingestion d'aliments secs et l'eau de boisson. La notion de palatabilité y est également présente.

Le rôle du sang comme vecteur de la nutrition du corps est nettement pressenti dans "Parties des animaux " et dans le traité " De l'âme ", on voit apparaître des « éléments de psychologie ». Trois âmes gouvernent le corps : la nutritive, la sensorielle et la pensante. Ces âmes ont des fonctions précises, indépendantes ou liées et hiérarchisées. L'âme nutritive est présente dans tous les êtres vivants, elle est chargée d'assurer les grandes fonctions essentielles de l'organisme. La sensation ou appétit dépend de l'âme sensorielle. Pour Aristote, l'appétit est défini comme le désir de l'agréable. Le désir peut porter sur l'eau ou sur des substances sèches et chaudes (I'aliment). Le livre II, 10 traite encore du goût et de la saveur des aliments. Les organes des sens ont une fonction d'analyse sensorielle destinée à la reconnaissance et au choix de l'aliment. On peut donc dire que leur rôle a été intuitivement découvert à cette époque, ou à une époque antérieure à Aristote (?).

Le traité " De la génération des animaux » nous éclaire sur les origines des différentes âmes. L'âme nutritive commune à l'homme et aux animaux est déjà présente dans l'embryon (Livre II,3). L'âme sensitive se développe aussi dans l'embryon mais, contrairement à la première, elle n'est pas apportée par le sperme. Quant à l'âme pensante, elle est d'essence divine.

On peut retenir de l'œuvre d'Aristote que : $1^{\circ}$ Le goût sert à reconnaître les aliments ; $2^{\circ}$ L'appétit est un désir de manger et correspond à un plaisir ; $3^{\circ}$ Les prises d'aliments solides et liquides sont liées ; $4^{\circ}$ Le sang est le vecteur de la nutrition du corps et celle-ci dépend de l'ingestion des aliments ; $5^{\circ}$ La nutrition (ingestion d'aliment) dépend d'une âme nutritive, d'une âme sensitive et (peutêtre) d'une âme intellectuelle.

Dans Livre XI-§74, Pline (dit l'Ancien, 23-79) pense que la voracité des loups, connue sous le nom de "lycorexie », est liée à " un transit rapide ». D'un point de vue physiologique, Pline considérait la rétention des aliments dans l'estomac comme une source de rassasiement. Le Livre II- $\S 128$ est consacré à des problèmes de " nutrition humaine » : I'étude des causes de l'obésité, liées à l'inaction du corps. Dans ce même chapitre il définit la faim comme un besoin impérieux.

Galien (130-200) introduit un concept nouveau : le pneuma. Chaque organe reçoit du sang nourricier, du sang pneumatisé, du pneuma psychique. Cette conception des "régulations du fonctionnement des organes " a eu un succès millénaire car, à travers Fernel (1655) elle existe encore au 18e siècle. L'apport de Galien en "physiologie expérimentale », au sens Bernardien, est fondamental. 
Bernard (1865) dans son "Introduction à l'étude de la médecine expérimentale » le cite à deux reprises et lui donne le titre de "Père de la Médecine expérimentale ». Galien a un esprit "scientifique " fondé sur des expériences de vivisection sur le porc, la chèvre et le mouton. II a sans doute réalisé la première expérience de choix alimentaire : il place dans une cave des jarres en terre contenant de I'huile, du vin ou du lait ; puis il introduit un chevreau nouveau-né, obtenu par césarienne et observe le choix de l'animal.

\section{Les temps modernes.}

\section{La période préscientifique.}

A la Renaissance, le fondateur du terme de physiologie, Fernel (1506-1558), médecin du Roi Henri II, hérite de la science grecque. L'œuvre de Fernel (traduite en français en 1655), de tradition Galénique, est divisée en livres. Au livre 5, il définit la faim comme une faculté appétitive ou, un appétit naturel dont le siège est situé dans le foie. II distingue ensuite, de ce naturel appétit, l'appétit sensitif qui est "le désir de manger et de friander ». Cet appétit naturel, sensitif, est sexuel et alimentaire. Comme Aristote, Fernel distingue un appétit nutritif lou " naturel appétit ") à mettre en parallèle avec l'" âme nutritive ", et un appétit commun, sensitif, à rapprocher de l'« âme sensorielle ". II manquait I'« âme pensante ", que Fernel définit comme un appétit raisonnable. " ... lequel désire avec raison ce qu'il appète et souhaite, qui obéit au conseil et à la volonté de l'esprit et de la raison... ".

$\mathrm{Au}$ "Siècle des Lumières " une véritable réflexion physiologique commence à apparaître.

Dans la troisième édition des "Primae lineae physiologiae » Haller (1769, 1777,1778 ) laisse supposer que la motivation de la prise alimentaire est la recherche du plaisir (précurseur de la théorie Hédonistique). La sensation de faim serait due à l'autostimulation des parois de l'estomac qui se touchent quand l'estomac est vide. Les contractions (?) de l'estomac formeraient des plis qui frottent sur les nerfs de l'estomac. Cette " explication " sera reprise par Blumenbach (1797) et sera encore évoquée par Cannon et Washburn (1912) et Carlson et al. (1912 à 1915).

Beaucoup d'autres théories mécanistes semblables avaient été proposées à la suite des découvertes de mécanique céleste, du magnétisme, de l'électricité, de l'acoustique. Querelle du subjectif contre l'objectif, "l'animal machine " défini par Descartes lui valut son exil. Hartley (1755) fait de la faim et de la satiété un phénomène oscillatoire. L'apport de Buffon est beaucoup plus naturaliste (1774, 1858). Il aborde la faim et l'appétit sous l'angle comportemental : c'est un pionnier de l'éthologie.

Les idées de l'Antiquité et de cette période seront encore colportées au 19 e siècle où elles sont exposées dans les cours de physiologistes comme Broussais (1822), Richerand (1801, 1807, 1811, 1823) et reprises dans les thèses de médecine : Leroux (1804), Lamotte (1804), Chaumas (1815), Bayle (1816) et Willien (1838). 


\section{La période expérimentale.}

Le $19^{e}$ siècle a vu naître la méthode expérimentale en physiologie avec Flourens, Magendie, Bernard, Vulpian... Les concepts de faim, de satiété, d'appétit vont se préciser grâce à l'expérimentation animale.

Le développement de la méthode scientifique en chimie (pesées précises depuis Lavoisier), puis l'apport en chimie organique de Liebig en Allemagne (1842), vont permettre cette physiologie scientifique (Fourcault, 1829). Déjà au 19 e siècle les physiologistes appliquent les découvertes techniques à l'étude de leur art. Par exemple : Roentgen découvre les rayons $X$ en 1895 et Cannon les utilise pour l'étude de la motricité de l'estomac en 1897-98.

\section{- Les théories mécanistes.}

Le début des recherches expérimentales sur la digestion et la prise alimentaire commence avec l'annonce d'un prix scientifique d'un montant de 3000 francs offert par l'Académie des Sciences en 1823 et dont les résultats devaient parvenir en 1825. Le sujet était : " de déterminer, par une série d'expériences chimiques et physiologiques, quels sont les phénomènes qui se succèdent dans les organes digestifs durant l'acte de la digestion $"$.

Deux équipes, au moins, ont entrepris des travaux expérimentaux pour ce concours. En France, il s'agit de Leuret et Lassaigne (1825) et en Allemagne de Tiedemann et Gmelin (1826).

Les auteurs français font une étude anatomique très précise de l'organisation du tractus digestif. A la page 75 , ils signalent que l'estomac est innervé par le pneumogastrique et le grand sympathique. II convient de noter que le pneumogastrique est à cette époque considéré comme la $8^{e}$ paire des nerfs crâniens. Ils remarquent que l'estomac est vide entre les repas. Leuret et Lassaigne observent alors le déclenchement de la sécrétion gastrique par l'arrivée des aliments. Ils font l'analyse chimique du suc gastrique recueilli dans plusieurs espèces (chien, crapaud, grenouille, lézard, canard) qui leur semblent représentatifs des quatre grandes classes de vertébrés. Ils estiment que l'acidité est due à la présence d'acide lactique.

En 1824 Prout avait isolé de l'acide chlorhydrique.

De même, Magendie cite encore l'acide lactique dans sa troisième édition du précis élémentaire de physiologie (1833) et il précise : " un habile chimiste anglais, M. W. Prout, a cru reconnaître dans l'acide du suc gastrique des animaux l'acide hydrochlorique libre, mais ses expériences sont contestées par $M$. Lassaigne (abrégé de chimie de 1829). II serait d'ailleurs extraordinaire qu'un corps aussi facile à reconnaître eût échappé à l'investigation de chimistes tels que Berzélius, Thénard, Chevreul " (cf. Ruckebusch, 1979 pour l'historique de la sécrétion gastrique).

Quel est le rôle du pneumogastrique dans la digestion ? II semble que les premières sections des pneumogastriques, en vue de l'étude de la digestion, doivent être attribuées à Baglivi, élève de Malpighi (vers 1700) [De telles sections ont été pratiquées par Rufus d'Ephèse, Galien vers 160 , puis A. Paré en 1614, mais avec des " hypothèses " différentes]. 
Le 23 juin 1825, en présence du Prof. Dupuy de l'école d'Alfort, Leuret et Lassaigne font la section et l'ablation de quelques centimètres des pneumogastriques au niveau du cou d'un cheval et la trachéotomie, pour éviter la suffocation. " II (le cheval) a continué de boire et de manger. " Le cheval est mort 6 jours après. A l'autopsie, Leuret et Lassaigne observent que l'œsophage est dilaté par les aliments, mais ils n'en tirent pas de conclusion. Nous avons ici la première démonstration expérimentale indiscutable que les nerfs pneumogastriques ne sont pas indispensables pour la prise alimentaire. En d'autres termes, pour parler comme les physiologistes de cette époque, la faim peut être perçue en l'absence des nerfs pneumogastriques qui proviennent de l'estomac.

Ce même mémoire de Leuret et Lassaigne est encore remarquable à un autre titre. En effet, en 1825, par conséquent 80 ans avant Bayliss et Starling, ils ont déjà mis en évidence le déclenchement de la sécrétion pancréatique après l'application d'eau vinaigrée sur le duodénum du chien (comme le ferait le chyme gastrique acide).

Le mémoire de Leuret et Lassaigne se termine par un appendice : « De la faim et de la soif. " " La faim consiste dans le besoin de prendre des aliments. On rapporte son siège à l'estomac ; cependant on ne sait pas encore quelle cause immédiate, résidant dans cet organe, peut la déterminer. " II est évident pour les auteurs que la sensation est transmise au cerveau : "Nous n'entrerons pas dans le détail des différentes hypothèses qui ont été émises sur la cause prochaine de la faim, parce qu'elles sont toutes insuffisantes... Nous examinerons seulement si l'on est fondé à regarder les nerfs de la huitième paire comme les organes destinés à transmettre au cerveau la sensation de la faim ». Nous connaissons déjà la réponse: "Les Chevaux... ont mangé comme auparavant et avec un appétit égal, au moins en apparence, à celui qu'ils avaient quand ils se portaient bien. "

De cette étude des travaux de Leuret et Lassaigne, on peut tirer les conclusions suivantes : $1^{\circ}$ Les auteurs sont de bons scientifiques et utilisent la méthode expérimentale avec rigueur ; $2^{\circ}$ Ils se trompent néanmoins quand ils pensent que l'acide sécrété par l'estomac est de l'acide lactique. Mais ils n'ont pas fait d'analyse poussée et ils sont influencés par les recherches de Berzélius, Thénard, Chevreul qui font autorité en la matière. Un excès de notoriété peut être un obstacle épistémologique : $3^{\circ}$ La section des pneumogastriques n'abolit pas la faim.

Le travail de Tiedmann et Gmelin est beaucoup plus complet que celui de Leuret et Lassaigne, les expériences sont très nombreuses, et l'analyse bibliographique très bien faite. Ils établissent que :

$1^{\circ}$ Le suc gastrique contient bien de l'acide chlorhydrique. Tiedemann et Gmelin reconnaissent l'antériorité de la découverte à l'Anglais Prout, bien que leur propre découverte soit exactement de la même période (février 1824).

$2^{\circ}$ L'estomac, observé sur des animaux sacrifiés après avoir été mis à jeun, est toujours resserré sur lui-même. Ce sont les aliments qui distendent les parois. L'organe change de forme générale et subit une légère rotation. Les aliments déclenchent la motricité. Les mouvements se propagent du cardia vers le pylore ; leur intensité semble dépendre de la nature des aliments. Les sécrétions gastriques sont également déclenchées par l'arrivée des aliments : à jeun, l'estomac est 
vide; elles sont plus abondantes si l'animal ingère des substances « difficiles à digérer " (nature protéique).

$3^{\circ}$ Les auteurs citent Rufus d'Ephèse comme étant le premier auteur à avoir étudié l'action de la section des pneumogastriques sur la digestion. Ils citent ensuite Baglivi.

$4^{\text {o }}$ Tiedemann et Gmelin ont sectionné, à la fois, les pneumogastriques et les nerfs grands sympathiques au niveau du cou du chien. Ces sections n'abolissent pas la prise alimentaire mais déclenchent presque toujours des vomissements. L'estomac est toujours vide dans ces expériences. En fait, les aliments ne peuvent pas franchir le cardia et restent dans l'oesophage. Ils sont vomis pour cette raison.

Ces deux mémoires, fondamentaux par certains résultats, ne proposent que des théories mécanistes de la faim. C'est encore le cas pour Adelon (1831) ou Blondlot (1843) et de Beaumont (1833, 1847). Ces théories s'opposeront aux théories humorales (cf. Serres, 1832 et Brachet, 1837).

\section{- Les théories humorales.}

Dans son "Précis élémentaire de physiologie » (1833) Magendie consacre un article à la faim. " Le besoin des aliments solides est caractérisé par un sentiment particulier dans la région de l'estomac et par une faiblesse générale plus ou moins marquée. »

Magendie introduit enfin une notion essentielle : c'est la souffrance générale de l'organisme qui est perçue et qui se traduit par un besoin. C'est la première apparition durant cette période de la notion que la faim n'est pas seulement une sensation mais un état de besoin dont la cause est un déficit de l'organisme et l'effet, la prise d'aliment qui réparera un déficit.

Magendie distingue les phénomènes locaux et les phénomènes généraux de la faim :

- Les phénomènes locaux seraient la conséquence d'un resserrement et de contractions de l'estomac. Il critique vigoureusement les hypothèses mécanistes : "... la plupart de ces données sont conjoncturales ». Puis il apporte ses expériences personnelles. Magendie remarque d'abord, qu'après 24, 48 ou 72 h d'abstinence complète, il n'y a pas de contractions ni de resserrement de l'estomac (alors qu'il y a déjà désir vraisemblable de prendre des aliments). L'estomac change d'aspect au $4^{e}$ ou $5^{e}$ jour. Pour Bichat, cité par Magendie, la pression intragastrique est identique si l'estomac est vide ou plein. Une expérience simple de Magendie (introduction d'un ballon dans l'estomac) montre que la pression intragastrique chute lors de l'abstinence : il y a un relâchement de l'estomac. Des observations systématiques lui prouvent aussi que l'estomac à jeun ne contient pas de bile : ce n'est donc pas un reflux de bile du duodénum vers l'estomac qui serait la cause de la faim. Ce n'est pas non plus le mucus qui tapisse l'estomac: si l'abstinence est prolongée, il y a d'autant moins de mucus. Comme nous l'avons déjà vu à de multiples occasions, on a tenté d'expliquer les phénomènes locaux de la faim par une redistribution de sang dans l'estomac, la rate, le foie. Magendie constate que lors de l'abstinence, l'apport de sang n'est pas seulement diminué dans l'estomac, mais que ce phénomène est valable pour tous les viscères. 
- Les phénomènes généraux traduisent un affaiblissement et une diminution de l'action de tous les organes et de toutes les fonctions. "On dit que l'absorption seule devient plus active, mais rien n'est rigoureusement démontré à cet égard. ")

L'appétit est un premier degré de la faim, il ne doit pas être confondu avec le choix des aliments. II fait donc partie des sentiments.

Quelles sont les causes rendant la faim plus intense ? «... en général toutes les causes qui mettent en jeu l'action des organes et accélèrent le mouvement nutritif, avec lequel la faim est essentiellement liée ". Même remarque que cidessus, Magendie rapporte l'intensité de la faim à l'intensité de la dépense.

Enfin, il s'interroge sur les causes prochaines de la faim ; après avoir rappelé les théories classiques il expose très clairement son avis : " La faim résulte, comme toutes les autres sensations internes, de l'action du système nerveux ; elle n'a d'autre siège que ce système lui-même, et d'autres causes que les lois de l'organisation. Ce qui prouve bien la vérité de cette assertion, c'est que (la faim) continue quelquefois, quoique l'estomac soit rempli d'aliments ; c'est qu'elle peut ne pas se développer, quoique l'estomac soit vide depuis longtemps ; enfin, c'est qu'elle est soumise à l'habitude, au point de cesser spontanément quand l'heure habituelle du repas est passée ").

Un peu plus tard, dans sa leçon du 20 décembre 1837, professée au Collège de France (publiée en 1839), ce concept de faim lié à une modification du sang est encore très nettement exposé. « ... voyons les effets que produit la privation des aliments solides. Si I'on supprime par degrés ou subitement la nourriture d'un animal, et qu'on examine son sang quelque temps après, on remarque que, sous l'influence de ce régime, certaines modifications se manifestent dans le liquide... Qu'est-ce que la faim, répéterai-je, sinon l'instinct sous peine de maladie et de mort à l'animal, de fournir à la reproduction du liquide sanguin ».

L'apport de celui qui fut le maître de Bernard est donc une étape décisive dans l'évolution des concepts. La conception humorale est d'ailleurs admise par certains de ses contemporains (Serres 1832/1835) qui s'opposent parfois aux tenants de la théorie mécanique (Brachet 1823, 1837 ; Brachet et Fouichoux, 1835). De grands physiologistes allemands comme Burdach (1837 à 1841) sont de l'avis de Magendie comme cela apparaît clairement à la lecture de son traité de physiologie, d'un intérêt exceptionnel.

Lorsque paraît la deuxième édition (1842) de ses " Recherches expérimentales sur les propriétés et les fonctions du système nerveux dans les animaux vertébrés. " Flourens a 48 ans (et Bernard 39).

Cet ouvrage est remarquable car il contient la première description du « centre respiratoire ", mais aussi, par un certain nombre d'expériences sur le cerveau des animaux vertébrés.

Flourens s'interroge sur les fonctions du cerveau proprement dit (hémisphères cérébraux). II réalise une série d'expériences d'ablations des deux hémisphères, chez la poule, en respectant les couches inférieures auxquelles les racines des bulbes olfactifs adhèrent. Elle devient sourde et aveugle, mais reste en vie pendant 6 mois. C'est donc une expérience très valable du fait de cette durée. Le surlendemain de l'opération, " on fait boire et manger l'animal ", " jamais, elle (la 
poule) n'a donné de signes de volonté manifeste ", " elle ne savait ni s'abriter ni manger d'elle-même ». "Vainement approchait-on la nourriture de son bec ou de ses narines; vainement la lui mettait-on dans le bout du bec : la poule n'odorait ni ne goûtait, ni n'avalait : la nourriture restait dans le bout du bec ». Placée au fond du bec, la nourriture était déglutie: "la déglutition ne demande pas de volonté. ")

Flourens tire, de l'ensemble de plusieurs expériences, les conclusions suivantes : $1^{\circ}$ les lobes cérébraux sont le siège de la perception sensorielle $; 2^{\circ}$ les lobes cérébraux contiennent les formes d'intelligence propre à chaque espèce ; $3^{\circ}$ les lobes cérébraux ont la faculté de percevoir et de vouloir en relation avec les facultés d'exciter et de coordonner ; $4^{\circ}$ l'ablation d'un seul lobe ne fait perdre la vue que d'un seul œil ; $5^{\circ}$ ceci est une loi générale chez les vertébrés ; $6^{\circ}$ la volonté d'ingérer dépend des hémisphères cérébraux. L'estomac seul est incapable de déclencher la prise alimentaire. [Mais on connaissait également à cette époque, le cas d'enfants anencéphales capables de " prendre " le sein (nous n'avons pas trouvé de précisions suffisantes dans les descriptions cliniques concernant la prise volontaire, ou non, du sein). Il est donc impossible de dire si un quelconque désir d'ingérer existe chez ces enfants sans hémisphères]. Quoi qu'il en soit, sur le plan anatomique, il y a sans doute de grandes différences entre les ablations chirurgicales et les anomalies pathologiques, et ce fait n'infirme en rien les conclusions de Flourens.

Ces conclusions, à très peu près, sont encore admises aujourd'hui.

Mais c'est par des expériences de section des nerfs pneumogastriques et surtout par la perfusion de "bouillons nutritifs " dans les veines que les théories humorales furent définitivement établies. A ce point de vue l'apport de Longet est fondamental.

Longet $(1849,1857,1869)$ pense que les nerfs vagues peuvent conduire des impressions en provenance de l'estomac : " mais est-ce réellement dans cet organe que siège la faim ? Non certainement... ", "... enfin on peut faire cesser le sentiment de faim, sans introduire d'aliments ni d'autres corps solides dans l'estomac, ainsi que le prouvent les injections de bouillon dans les veines ". Longet a-t-il effectué cette dernière expérience? Comme le rappelle Ruckebusch (1979), il est de tradition au $16^{\text {e }}$ siècle de faire des perfusions de sang de chiens (espèce vile) au cheval (espèce noble) et de décrire la "satiété " chez le cheval perfusé... II est également possible que Longet fasse allusion ici aux travaux de Schiff (1867) qui a alimenté des animaux par perfusion parentérale.

« La faim est l'expression d'un état général qui se traduit par une sensation spéciale que nous rapportons à l'endroit où elle se fait sentir, bien qu'en réalité, elle ne siège pas uniquement en cet endroit. " Puis, cette phrase, très convaincante par sa justesse et son humour : " II en est d'ailleurs d'autres sensations internes: le besoin de dormir se manifeste par une sensation particulière aux yeux, aux paupières, et certes il ne viendra à l'esprit de personne de localiser le sommeil dans les yeux »... « C'est donc dans l'organisme, en général, qu'il faut placer le sentiment de la faim et la sensation particulière éprouvée dans la région épigastrique doit être considérée comme une manifestation limitée d'un état général, comme le prodrome des nombreux phénomènes de la faim. " 
Quelle est la structure capable d'intégrer la sensation ? " Après section des pneumogastriques les chiens peuvent se remettre à manger sans problème. II est donc apparu à un certain nombre d'auteurs que dans ce cas les chiens ingèrent pour satisfaire leur sensualité gustative. " "Toutefois, j'ai tenté plusieurs expériences qui me font croire que le goût n'est pas ici le principal mobile : en effet, quelques chiens qui avaient subi, de chaque côté, la section des nerfs glossopharyngiens et linguaux et celle des pneumogastriques, ont mangé sans dégoût, en assez notable quantité, des substances alimentaires ramollies dans une décoction légère de coloquinte. " Donc, chez le chien agueusique et sans nerfs pneumogastriques, la faim est encore perçue.

L'apport de Longet est original et considérable et ne sera pas infirmé par Bernard.

Dans l'œuvre scientifique de Bernard, il y a peu de documents relatifs au concept de faim ; dans ses leçons de 1858 il précise : "On avait présenté l'estomac comme le siège de la sensation de la faim. II n'en est rien : après l'opération (bivagotomie), les animaux continuent à manger surtout lorsqu'ils ont été opérés étant à jeun." "

Grâce au « catalogue des manuscrits de Claude Bernard " (Grmek 1967), nous avons pu consulter un certain nombre de travaux inédits :

- Le manuscrit C VIII $n^{\circ} 5$ s'intitule "Notes sur la digestion ". C'est vraisemblablement le projet d'un cours : " Le 22 mai 1849. Plan à suivre dans l'exposé des phénomènes de la digestion. 23 mai 1849. Digestion... Faim, soif... insalivation. » "Deux sensations spéciales avertissent l'homme et les animaux du besoin d'introduire dans le canal intestinal des aliments solides et liquides. Ce sont la faim et la soif. Des appétits spéciaux, des goûts particuliers existent aussi pour certains aliments (appétit) et déterminent, suivant leur nature, les animaux à se nourrir de telle ou telle substance alimentaire. Un vautour mourut de faim à côté d'un tas de blé (Réaumur). Cependant cet instinct peut être raisonné. Du blé concassé nourrirait un vautour. Dans l'état ordinaire la faim et la soif sont en rapport avec les besoins de l'organisme et disparaissent quand l'estomac est plein. Excepté boulimie, fringale, cas morbide et pica, goût altéré. La faim et la soif tiennent à un besoin général de l'organisme localisé dans le système nerveux. Toutefois, le phénomène ne cesse pas avant que l'organisme soit réparé. Chiens fistulés buvant toujours, de même que chevaux à œesophage coupé. Faim et soif apaisés par injections dans les veines. "

C'est donc le besoin de boire et de manger qui détermine la fonction. Les concepts de faim et d'appétit selon Bernard (en 1849) peuvent donc être résumés ainsi : $1^{\circ}$ La faim est un besoin (le besoin de manger) ; $2^{\circ} C^{\prime}$ est un besoin général de l'organisme ; $3^{\circ}$ II est localisé dans le système nerveux ; $4^{\circ} \|$.ne cesse que si le déficit est réparé, $5^{\circ}$ ॥ existe des appétits spéciaux qui permettent à l'animal de choisir une nourriture conforme à son espèce. Cet apport n'est pas original (en ce sens qu'il ne repose pas sur des faits expérimentaux originaux), si ce n'est qu'il provient d'un scientifique de très grande valeur et qu'on commence à entrevoir le concept d'homéostasie, notamment, quand Bernard pense que la faim est un besoin général qui ne cessera que dans la mesure où le déficit est réparé.

Puis Bernard redéfinit la faim : comme " un phénomène physiologique qui 
provoque l'introduction des substances alimentaires dans l'intestin et leur transport de la bouche dans l'estomac ". II n'y a pas de doute, la faim apparaît ici clairement comme la motivation déclenchante de la prise alimentaire. C'est cette notion qui prévaudra jusqu'à nous.

- Manuscrit 7a (C VIII e $n^{\circ} 7 a$ ). Le dimanche 10 décembre 1843, Bernard a effectué la section des deux pneumogastriques sur un chien portant une large fistule de l'estomac (préalablement réalisée au mois d'octobre 1843). II observe l'effet de la section des nerfs pneumogastriques en rapport avec le transit œsophagien et les sécrétions gastriques : «Aussitôt après la section des pneumogastriques, je donne à manger au chien une soupe au lait sucrée. II la mange avec peine et fait beaucoup d'efforts pour avaler ; ... " Dix pages après, il conclut : « après la section des pneumogastriques, le bol alimentaire ne peut donc plus arriver dans l'estomac, pourquoi s'arrête-t-il au-dessus du diaphragme, est-ce qu'il y a là une obstruction, un resserrement qui empêche les aliments de descendre ?".

L'apport de Bernard, bien qu'il n'ait pas directement étudié le déterminisme de la prise alimentaire, est fondamental par les précisions claires qu'il nous donne sur les rôles de l'estomac et des pneumogastriques.

Nous avons également recherché l'opinion des physiologistes qui n'ont pas étudié expérimentalement la faim. Parmi eux, citons Colin (1854), Béclard (1859), Bérard (1848-1851), Béraud (1853), Carlet (1871), Bardier (1904), Laulanié (1905), Gley (1913). Ils n'apportent pas d'éléments nouveaux, ni psychologiques, ni médicaux.

En résumé, à la fin du 19 e siècle, grâce aux physiologistes, peu à peu, on commence à entrevoir une théorie "centrale " de la faim ; mais il faudra encore attendre 1940 pour que cette idée fasse l'objet d'une étude expérimentale rigoureuse.

On peut se demander pourquoi, pendant si longtemps, les physiologistes ont voulu faire de la faim une sensation purement gastrique. Béclard nous éclaire sur ce point : «C'est parce que beaucoup de physiologistes comparent, à tort, les sensations de la faim avec celles des organes des sens, que l'on a cherché à fixer son siège organique, ainsi que le nerf chargé de transmettre à l'encéphale, ses impressions locales. " On pourrait ajouter que si les physiologistes ont si longtemps hésité, et même jusqu'en 1965, c'est qu'ils ont confondu un "sentiment " (la faim) et un acte physiologique : la prise alimentaire.

\section{logues.}

- Essais de localisation cérébrale d'un centre de la faim : l'apport des neuro-

Les expériences que nous avons citées, notamment celles relatives à la section des nerfs pneumogastriques, ont incité les physiologistes à rechercher dans le cerveau, des centres de la prise alimentaire. Sans doute, stimulés par les publications de Gall (1822-1825) sur les localisations cérébrales, plus peutêtre pour les infirmer que pour les confirmer, des neurologues de renom comme Flourens, Ferrier, Charcot, Pitres, Vulpian, Luys et Goltz ont établi les liens entre des modifications de la prise alimentaire et des lésions cérébrales. Certains d'entre eux ont pratiqué des opérations chirurgicales, sur l'animal, pour vérifier les hypothèses posées lors d'examen de cas de pathologie humaine. 
Flourens, en 1842, donne la première publication du " centre respiratoire " : le nœud vital et l'ouvrage est également remarquable par un certain nombre d'expériences d'ablations des deux hémisphères sur les Oiseaux. L'ablation respecte les couches inférieures de ces lobes (la poule devient sourde et aveugle, mais reste en vie pendant 6 mois).

Ferrier (1878-1880), dans ses ouvrages, nous expose une série de lobectomies, réalisées sur le singe. II pense ainsi obtenir par la soudaineté de l'intervention, comparée à une lente dégénérescence de la maladie, une perturbation plus facile à analyser : " L'ablation... des lobes préfrontaux n'est suivie d'aucun résultat physiologique défini. Les animaux conservent leurs appétits et leurs instincts et sont susceptibles de faire preuve d'émotion "... « II est parfaitement évident, d'après les résultats de la physiologie et de la clinique, que les hémisphères cérébraux sont le siège de la sensation, ou mieux, de la perception des sensations, pour éviter toute discussion de mots $»$.

Ferrier considère la faim comme une sensation, et dans ce cas, elle serait perçue ou/et analysée au niveau cortical : " ... j'observai de même que, contrairement à ce qui se passe habituellement lors de lésions destructives d'autres parties des hémisphères, les animaux refusaient de manger, ce que je regarde un peu comme le résultat de cette lésion, d'après mes nombreuses et attentives observations des effets de la destruction de toutes les parties du cerveau "... " J'admets volontiers que ma conclusion est quelque peu affaiblie par le fait que, chez un des animaux auxquels j'avais enlevé les deux lobes occipitaux l'appétit revint après cinq jours d'abstinence..."

Cette localisation des centres de la faim dans les lobes occipitaux a été infirmée par la suite, mais cette erreur a été longtemps reprochée à Ferrier. On peut pourtant dire, à sa décharge, qu'il était particulièrement prudent dans ses affirmations et en demandant une confirmation ou une infirmation ultérieure de son hypothèse...

Beaucoup d'autres neurologues ont recherché la situation anatomique des centres de la faim.

Comme le rapporte Bardier (1904) : Combes, Spurzheim et Broussais admettent un " organe de l'alimentivité " qui serait placé dans les fosses latérales et moyennes de la base du crâne. "Rosenthal suppose l'existence d'un centre qu'il appelle " centre de la faim ". Pour Paget, cette structure corticale, serait placée à l'extrémité antérieure du lobe temporo-sphénoïdal près des centres du langage ".

A la même époque, comme l'indique Soury (1899), l'école de Strasbourg avec Goltz et son élève Schrader ont réalisé des lobectomies corticales dans diverses espèces. Schrader signale que des grenouilles dont les hémisphères cérébraux sont détruits sont encore capables de capturer et d'avaler des mouches. Chez les pigeons, sans hémisphères cérébraux, Schrader remarque qu'il faut leur placer l'aliment dans le bec pour qu'ils le mangent. Par contre, Goltz a pu enlever les hémisphères cérébraux chez un chien sans abolir la prise alimentaire. Ce chien vécut 9 mois : "Ainsi ce chien sans cerveau acquit de nouveau la faculté de manger et de boire lui-même ". L'autopsie, faite par Schrader révèle que le cortex avait été enlevé ainsi que les corps striés et une partie des tubercules quadrijumeaux. II restait donc la base du cerveau, le pont de Varole et le bulbe rachidien. 
Ces expériences démonstratives mais peu reproductibles laissaient donc supposer que les "centres de la faim » étaient situés à la base du cerveau.

Deux grands neurologues, Vulpian (1866) et Luys (1876) précisent encore ces localisations. Dans ses leçons de 1866 Vulpian nous parle de l'instinct et de l'intelligence : "Parmi les instincts relatifs à la conservation de l'individu, il faut inscrire celui qui préside au choix des aliments, qui naît avec l'animal et subit, lorsqu'il le faut, des variations en rapport avec les métamorphoses ". Ces instincts sont, d'après Vulpian, placés dans les lobes cérébraux. Les expériences de Flourens et des expériences d'ablation systématique des différents étages du cerveau lui permettent de l'affirmer.

Luys (1876) nous apporte des éléments nouveaux : il s'interroge sur l'ontogénie de la sensibilité. Il admet que l'enfant qui vient de naître agit automatiquement : "A partir de ce premier moment, l'enfant prend le sein de la nourrice automatiquement et en vertu des forces vives héréditaires qui existent déjà à l'état latent dans son système nerveux ". Les "forces vives " (ou instincts) sont, d'après Luys, " héritées ". II y a donc dans le cerveau une structure qui va permettre à l'enfant, mis en présence d'un sein d'ingérer du lait. Mais par la suite, des phénomènes d'apprentissage vont se surajouter : " son sensorium est ému ". Ce qui signifie que sa perception : consciente, intelligente est analysée et cette analyse sera mémorisée. Pour Luys, la mémoire est une " phosphorescence " des cellules et il admet des localisations corticales séparées pour les différentes mémoires sensorielles. Ces mémoires vont permettre des acquis qui peu à peu vont transformer la prise alimentaire en gastronomie...

\section{- A la recherche d'un centre alimentaire.}

Nous avons utilisé un document assez tardif, sur l'existence d'un " centre alimentaire », que Pawlow (1921) nomme " le centre alimentaire ». Pawlow affirme la réalité d'une telle structure qu'il compare au centre respiratoire (nœud vital de Flourens) : " II est clair que le premier stimulant de ce centre... est dans la composition chimique du sang de l'animal qui n'a pas mangé depuis un certain nombre d'heures, et dont le sang, par conséquent, est " affamé ». "

Le fonctionnement du centre n'est pas toujours subordonné à une activité sensorielle réflexe : « ... je sectionnais les nerfs vagues, les deux paires des nerfs récepteurs de la langue et malgré cela, les animaux se sentaient vis-à-vis de l'aliment comme des animaux normaux ».

Pour la première fois, un auteur admet que le centre alimentaire puisse être non seulement activé, mais aussi inhibé "comme le centre respiratoire ». Suit une expérience et sa conclusion : « ... tuer l'appétit en mangeant un peu avant d'aller à table ».

Enfin, Pawlow s'interroge sur l'organisation et la situation hypothétiques du centre alimentaire : "II est certainement très complexe, en plusieurs parties distinctes, c'est certainement un centre récepteur, constitué de neurones récepteurs, puisqu'il reçoit des excitations diverses, tant intérieures que réflexe ». Où est-il situé ? Impossible à dire mais « il faut admettre qu'il occupe différents étages du système nerveux central ". Pawlow prend l'exemple du pigeon décérébré qui 
avale si on lui place le grain dans le bec : " donc, une partie du centre alimentaire se trouve au-dessous des hémisphères cérébraux ". "Mais il y a certainement d'autres parties situées dans les hémisphères. Ce peuvent être par exemple les centres gustatifs... ».

\section{Conclusions.}

Ces travaux ne sont plus cités aujourd'hui mais gardent toute leur valeur historique et épistémologique.

Le développement des techniques de chirurgie stéréotaxique à partir des années 1906 avec Horsley et Clarke, la meilleure connaissance de la neuroanatomie et de la neurohistologie, les progrès en électronique médicale vers 1940, permirent d'aborder l'étude du système nerveux central avec précision. (Cf. Le Magnen, 1986. Ibid.). Deux découvertes fondamentales eurent lieu à cette époque : celle de l'hyperphagie induite par la lésion médio-ventrale de l'hypothalamus (Heterington et Ranson, 1940) et celle de l'aphagie induite par la lésion latérale de I'hypothalamus (Anand et Brobeck, 1952). Les travaux se poursuivirent par la recherche des facteurs métaboliques ou/et nerveux capables de réguler l'ingestion alimentaire. Des théories glucostatique, lipostatique, aminoacidémique, thermostatique virent le jour. Les facteurs de régulation d'origine périphérique comme l'encombrement de la ration ou la vitesse du transit digestif furent étudiés. Chez les Ruminants, les produits particuliers libérés par les actions microbiennes dans le rumen comme les acides gras volatils, furent pressentis comme facteurs de rassasiement à court terme (Le Bars et al., 1954). Les recherches sur la motricité des estomacs, le rôle de l'encombrement de la ration, les relations entre les repas et la vigilance furent développées par Ruckebusch (1963).

Aucune de ces hypothèses n'a pu être entièrement vérifiée. Comme le souligne Focant (1986), chez les petits Ruminants, « il semble en effet que le contrôle de la prise alimentaire soit plurifactoriel avec intégration centrale ".

Les recherches de neurophysiologie sur le système nerveux central sont essentiellement avancées chez le Rat de laboratoire. La multiplicité des espèces animales à étudier, le peu de moyens humains et financiers consacrés aux recherches sur la prise alimentaire chez les animaux d'élevage sont partiellement responsables de notre ignorance.

2emes Journées sur la Nutrition et I'Alimentation des Herbivores, I.N.R.A., Paris, 19 et 20 mars 1986

\section{Références}

Sources : FMD : Bibliothèque Fac. Méd. Paris ; MU : Museum Hist. Nat. Paris ; CFD : Collège de France ; BN : Bibliothèque Nationale; ENVA: Ecole Vét. Alfort: INA: Inst. Nat. Agro. ParisGrignon ; AM : Académie de Médecine ; FG : Coll. particulière.

ADELON N. P., 1831. Physiologie de /'homme. Crochard, Paris. 2e Ed., 2, 551 pp. FG.

ANAND B. K., BROBECK J. R., 1952. Hypothalamic control of food intake in rats and cats. Yale J. Biol. Med., 24, 123-146.

ARISTOTE (384-322 av. J. C.), vers 347-342 av. J. C. De l'âme. Texe de A. Jannone. Trad. E. Barbotin. Ed. Les Belles Lettres, Paris, 1980. FG 
ARISTOTE, vers 347-342 av. J. C. Histoire des animaux. Texte et traduction de Pierre Louis. Ed, Les Belles Lettres, Paris. 10 livres. 1964. FG.

ARISTOTE, vers 347 et 342 av. J. C. Parties des animaux. Traduction et texte de Pierre Louis. Ed. Les Belles Lettres, Paris. 1956. FG.

BARDIER E., 1904. Faim. In Dictionnaire physiologique de Charles Richet. Félix Alcan, Paris. 6, 1-29. FG.

BAYLE F. A., 1816. Considérations physiologiques et pathologiques sur la faim. Thèse de médecine. Paris. 37 pp. FMD.

BEAUMONT W., 1833. Experiments and observations on the gastric juice and the physiology of digestion. F.D. Allen, Plattsburgh. in $8^{\circ} .280$ pp. FMD 45029.

BEAUMONT W., 1847. Physiology of digestion; with experiment on the gastric juice. $2^{\circ}$ Ed. Burlington. p. 104. Ed. Goodrid, in $8^{\circ} .303$ pp. FMD 37272 et 45029.

BÉCLARD J., 1859. Traité de physiologie humaine, comprenant les principales notions de la physio-

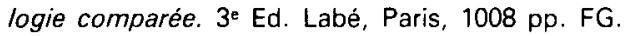

BÉRARD P. H., 1848-1851. Cours de physiologie. Ed. Labé, Paris. 3 vol. in $8^{\circ}$. FMD 35534 et 9535.

BÉRAUD B. J., 1853. Manuel de physiologie de l'homme et des principaux vertébrés... revu par M. Ch. Robin. Ed. Germer Baillière, Paris. VIII + 904 pp. MU 11970.

BERNARD Cl., 1841-1842. Expériences sur le système nerveux. Collège de France, Paris. Archives. Manuscrit. Cahier $n^{\circ}$ 1. CDF.

BERNARD Cl., 1843. Expériences sur la déglutition et l'insalivation du cheval. Collège de France, Paris. Archives. Manuscrit C VIII e, $n^{\circ} 7$ a. p. 172-174-184-194. CDF.

BERNARD Cl., 1847-1848. Physiologie expérimentale. Collège de France, Paris. Archives. Manuscrit VIII e, $n^{\circ} 7$ c. Cahier $n^{\circ} 4,102-104$. CDF.

BERNARD Cl., 1850-1860. Cahier de Notes. Edition du Cahier Rouge, par M. D. Grmek. Ed. Gallimard, Paris. 1965. 315 pp. FG.

BERNARD Cl., non daté. Suivre l'aliment. Faim. Soif. Collège de France, Paris. Archives. Manuscrit VIII e, $n^{\circ}$ 5. 1, 101-111. CDF.

BERNARD Cl., non daté. Analyses digestion. Faim. Soif. Collège de France, Paris. Archives. Manuscrit VIII e, no 13 b. p. 3-5 et 219-234. CDF.

BERNARD $\mathrm{Cl}$., non daté. Projets de mémoires de communications diverses. Médecine expérimentale. Physiologie générale. Collège de France, Paris. Archives. Cahier $n^{\circ} 3$. Manuscrit $1 \mathrm{~d}$. Page 69. CDF.

BERNARD Cl., 1858. Leçons sur la physiologie et la pathologie du système nerveux. Ed. J. B. Baillière, Paris. 2 tomes. $520+560$ pp. FG.

BERNARD Cl., 1865. Introduction à l'étude de la médecine expérimentale. Baillière, Paris. FG

BICHAT X., 1805. Recherches physiologiques sur la vie et la mort. 3 e Ed. Brosson et Gabon, Paris. 347 pp. FG.

BLONDLOT N., 1843. Traité analytique de la digestion considérée principalement dans l'homme et dans les animaux vertébrés. Grimblot, Raybois et Cie, Nancy. 471 pp. FG.

BLUMENBACH J. F., 1797. Institutions physiologiques, traduites du latin et augmentées de notes. Par J. F. Pugnet, J. T. Reymann et Cie, Lyon, pp. VIII + 328. MU. 216717.

BORDENAVE T., 1787. Essai sur la physiologie ou physique du corps humain. 4e Ed. Méquignon, Paris. 2 tomes. XIV + 293. IV + 374. MU Ch 853.

BRACHET J. L., 1823. Mémoire sur les fonctions du système nerveux ganglionnaire. Gabon et Lyon, Paris. 96 pp. Exemplaire de Cuvier. MU KK/100 Bz.

BRACHET J. L., 1837. Recherches expérimentales sur les fonctions du système nerveux ganglionnaire. Ed. Germer Baillière, Paris. 500 pp. Exemplaire de $\mathrm{Cl}$. Bernard. FG.

BRILLAT-SAVARIN A., 1838. Physiologie du goût ou méditations de gastronomie transcendante... suivi de la gastronomie, poème en quatre chants par Berchoux. Charpentier, Paris. in $8^{\circ}$. VIII $+493 \mathrm{pp}$. FMD 71289.

BRoUSSAIS F. J. V., 1822-1823. Traité de physiologie appliquée à la pathologie. Mlle Delaunay, Paris. 2 vol. in $8^{\circ} .361+575$ pp. MU 3897.

BRUNEMEIER E. H., CARLSON A. J., 1914. Contributions to the physiology of the stomach. XIX. Reflexes from the intestinal mucosa to the stomach. Am. J. Physiol., 36 (2), 191-195. MU PR 742. 
BURDACH C. F., 1837 à 1841. Traité de physiologie considérée comme science d'observation, avec des additions de MM. les professeurs Baer, Meyen, J. Muller, Rathke, Valentin, Wagner. Traduit de l'allemand par A. J. L. Jourdan. Ed. J. B. Baillière, Paris. 9 vol. in $\mathbf{8}^{\circ}$. FG.

CANNON W. B., WASHBURN A. L., 1911 et suiv. An explanation of hunger. Amer. J. Physiol., 29, 441-454. MU PR 742.

CANNON W. B., 1897. Movements of the alimentary canal. Présenté par H. P. Bowditch. Science, June 11, 1897. Vol. V, $n^{\circ}$ 128. 901-902. MU PR 506.

CANNON W. B., 1898. The movements of the stomach studied by means of the Röntgen rays. Amer. J. Physiol., 1, 359-382. MU PR 742.

CARLSON A. J., 1912. Contribution to the physiology of the stomach. I. The character of the movements of the empty stomach. Amer. J. Physiol., 31, 151-168. MU PR 742.

CARLSON A. J., 1912. Contribution to the physiology of the stomach. II. The relation between the contractions of the empty stomach and the sensation of hunger. Amer. J. Physiol., 31, 174-192. MU PR 742.

CARLSON A. J., 1912. Contribution to the physiology of the stomach. III. The contractions of the empty stomach inhibited reflexly from the mouth. Amer. J. Physiol., 31, 212-222. MU PR 742.

CARLSON A. J., 1912. Contribution to the physiology of the stomach. IV. The influence of the contractions of the empty stomach in man on the heart beat, and on the reflex excitability of the spinal cord. Amer. J. Physiol., 31, 318-327. MU PR 742.

CARLSON A. J., GINSBURG H., 1915. Contribution to the physiology of the stomach. XXIV. The tonus and hunger contractions of the stomach of the new-born. Amer. J. Physiol. 38, 29-32. MU PR 742.

CHARCOT J. M., PITRES A., 1980. Nouvelle contribution à l'étude des localisations motrices dans l'écorce des hémisphères du cerveau. Imprimé à la suite d'une traduction de D. Ferrier. Ed. Germer Baillière, Paris. 218-288. FG.

CARLET G., 1877. Faim et soif. Dictionnaire encyclopédique des Sciences médiacles. Dir. A. Dechambre, (4) 1, 155-160. Ed. Asselin et Masson, Paris. FG.

CHAUMAS J.-B., 1815. Considérations sur la faim. These Fac. Mèd. Paris. 19 pp. FMD.

COLIN G., 1854. Traité de Physiologie comparée des animaux domestiques. Ed. J. B. Baillière, Paris. 2 tomes in $8^{\circ} .607+628$ pp. FG.

DARWIN Ch., 1877. L'expression des émotions chez l'Homme et les animaux. Traduit de l'anglais par J. Pozzi et R. Benoit sur la $2^{\circ}$ Ed. C. Reinwald et Cie, Paris. in $8^{\circ} .404$ pp. MU 4303.

DUMAS Ch. L., 1800. Principes de physiologie ou introduction à la Science expérimentale, philosophique et médicale de L'homme vivant. Crapelet, Paris. 1, 480 pp. ENVA.

DESCARTES R., 1824-1826. L'homme. OEuvres de Descartes publiées par V. Cousin. 11 vol. Levrault, Paris. 1824-1826. MU 7121.

ENCYCLOPÉDIE INTERNATIONALE DES SCIENCES ET DES TECHNIQUES. 1971. Dir. Scientifique P. Auger et M. D. Grmek. Presses de la Cité, Paris. 10 vol. in Fo. Article Faim, 575-576. INA-PG.

FERNEL J., 1655. Les sept livres de la physiologie. Composez en latin, par messire Jean Fernel, premier médecin du Roi Henri II. Traduits en François par Charles de Saint Germain. Escuyer, Docteur en la faculté de médecine, conseiller et médecin ordinaire du Roy. Parisien. Jean Guignard le Jeune, Paris. FMD 33393 et ENVA.

FERRIER D., 1878. Les fonctions du cerveau. Traduit de l'anglais par C. de Varigny sur l'édition originale de 1876. Ed. Germer Baillière, Paris. 519 pp. FG.

FERRIER D., 1980. De la localisation des maladies cérébrales. Traduit de l'anglais par C. de Varigny. Suivi d'un mémoire sur les localisations motrices dans l'écorce des hémisphères du cerveau. Par MM. J. M. Charcot et A. Pitres. Ed. Germer Baillière, Paris. 288 pp. FG.

FLOURENS P., 1842. Recherches expérimentales sur les propriétés et les fonctions du système nerveux dans les animaux vertébrés. $2^{\circ}$ Ed. Ed. J. B. Baillière, Paris. 516 pp. FG. VIII + 101 pp. MU 119296 ou FG.

FLOURENS P., 1844. Mémoires d'anatomie et de physiologie comparée. Ed. J. B. Baillière, Paris.

FOCANT M., 1986. Contribution à l'étude du comportement alimentaire des petits Ruminants : rôle des acides gras volatils. Louvain-la-Neuve. Thèse Sci. agronom., 1-262. 
FOURCAULT A., 1829. Lois de l'organisme vivant, ou application des lois physico-chimiques à la physiologie. Rouen Fres, Paris. 2 tomes in $8^{\circ}$. XIV $-440+532$. MU 217166.

GALIEN (130-200), (cf. HÉGER). Dictionnaire physiologique de Charles Richet. F. Alcan, Paris. 6, 928-950. FG.

GALL F. J., 1822-1825. Sur les fonctions du cerveau et sur celles de chacune de ses parties. Chez l'auteur, Paris. 6 vol. in $8^{\circ}$. Tome I, Sur l'origine des qualités morales. Tome IV-V, Organologie ou exposition des instincts. MU 12176.

GALLOUIN F., FOCANT M., 1980. Bases physiologiques du comportement alimentaire des Ruminants. Reprod. Nutr. Dév., 20, 1563-1614.

GLEY E., 1913. Traité élémentaire de physiologie. Paris. J. B. Baillière. 17219.

GRMEK M. D., 1965. Claude Bernard. Cahier de Notes. Ed. Gallimard, Paris. 1-315. FG.

GRMEK M. D., 1967. Catalogue des manuscrits de Claude Bernard. Ed. Masson, Paris. 1-419. FG.

HALLER A. von, 1769. Eléments de physiologie. Trad. nouvelle du Latin en Français par M. Bordenave. Guileyn, Paris. 1 vol. in $12^{\circ} .1^{\text {re }}$ partie : 1-261: $2^{\circ}$ partie : 1-309. MU 217331 et Ch 851 .

HALLER A., 1777. Primae lineae physiologiae. Venetiis, 3e Ed. 1-330. FG.

HALLER A., 1778. De Partium Corporis Humane praecipuarum fabrica et functionibus. Opus quinquaginte annorum. Ex Prelis Societatum typographicarum, Bernae et Lausanae. Dédicacé à Spallanzani. 8 volumes ? FG.

HARTLEY D., 1755. Explication physique des gens, des idees et des mouvements tant volontaires qu'involontaires. Trad. de l'Anglais par M. l'Abbé Juraic. Delaistre, Godet, Reims. 2 vol. XXXVI + 502 pp. VIII + 474 pp. MU 217332 ou 217342.

HEDON E., 1904. Précis de physiologie, 4e Ed. Corrigée et augmentée. O. Dorin, Paris. II, 666 pp. 78-79. MU 217225.

HEEGER P., 1904. Galien, In Dictionnaire physiologique de Charles Richet. Ed. F. Alcan, Paris. Tome 6, 928-950. FG.

HETERINGTON A. W., RANSON S. W., 1940. Hypothalamic lesions and adiposity in the rat. Anat. Record., 78, 149-172.

HIPPOCRATE, -400 av. J. C. Du Régime. Ed. Les Belles Lettres, Paris. Trad. R. Joly. 1967. 1-141. FG.

JARRIGE R., 1986. REBISCHUNG J., 1950. Communication personnelle.

LAMOTTE J. A., 1804. Propositions sur la faim. Thèse Méd., Paris. 1-12. FMD.

LASSAIGNE J. L., 1829. Abrégé élémentaire de chimie considérée comme Science accesssoire à l'étude de la médecine, de la pharmacie et de l'histoire naturelle. Bechet jeune, Paris. 2 vol. in $8^{\circ}$. Tableaux et atlas. pp. XIX, 1160. MU 11302.

LAULANIÉ F., 1905. Eléments de physiologie. Ed. Asselin et Houzeau, Paris. 20 éd. 1-1214. FG.

LE BARS H., LEBRUMENT M., NITESCU R., SIMONNET H., 1954 . Recherches sur la motricité du rumen chez les petits Ruminants. 4. Action de l'injection intraveineuse d'acides gras à courte chaîne. Bull. Acad. vét., 27, 287.

LERŌUX J. L., 1804. Dissertation sur l'appétit et la faim. Thèse Méd., Paris. 1-31. FMD.

LEURET F., LASSAIGNE J. L., 1825. Recherches physiologiques et chimiques pour servir à I'histoire de la digestion. Ed. Mme Huzard, Paris. 1-217. FMD.

LE MAGNEN J., 1986. Hunger. Cambridge Univ. Press.

LITTRE E., 1876. Dictionnaire de la langue Française. Ed. Lib. Hachette et Cie. 1876. INA-PG.

LONGET F. A., 1849. Mémoire sur la véritable nature des nerfs pneumogastriques et les usages de leurs anastomoses. Riguoux Imp., Paris. in $8^{\circ}$. $36 \mathrm{pp}$. Extr. Arch. Générales de Médecine. MU $8^{\circ} \mathrm{BN} 155$ et $\mathrm{KK} / 73 \mathrm{c}$.

LONGET F. A., 1857. Traité de physiologie. Ed. V. Masson, 1, 1-682. FG.

LONGET F. A., 1869. Traité de physiologie. 3e Ed. Ed. Germer Baillière, Paris. 3, 1-1010. FG.

LUYS, J., 1876. Le cerveau et ses fonctions. Ed. Germer Baillière, Paris. 1-260. FG.

MAGENDIE F., 1809. Mémoire sur les organes de l'absorptıon chez les Mammifères, Paris. in $8^{\circ}$. 16 pp. Exemplaire de Cuvier. KK/220d.

MAGENDIE F:, 1813. Mémoire sur le vomissement, lu à la première classe de l'Institut de France par F. Magendie, Docteur-Médecin de la Faculté de Paris, Prospecteur à la même faculté, Professeur d'anatomie et de physiologie, suivi du rapport fait à la classe par MM. Cuvier, Pinel et Percy. Ed. Crochard, Paris. 48 pp. MU 12720 (5). 
MAGENDIE F., 1815. Trad. E. Home, Observations sur les fonctions. S.C. in $8^{\circ}$. MU 12720 (3)

MAGENDIE F., 1816-1817. Précis élémentaire de physiologie, tome II (11). Ed. Méquignon-Marvis, Paris. 1816-1817. 2 tomes en 1 vol. in $8^{\circ}$. MU 3816 1-96.

MAGENDIE F., 1823. Mémoire sur quelques découvertes récentes relatives aux fonctions du système nerveux. Ed. Méquignon-Marvis, Paris. in $8^{\circ} .25$ pp. MU 12720 (1).

MAGENDIE F., 1824. Mémoires sur les fonctions de quelques parties du système nerveux. J. Physiol. Exp. Patho. de F. Magendie. Ed. I'auteur et Méquignon-Marvis, Paris. Tome 4. 399-407. MU PR 4129.

MAGENDIE F., 1821-1829-1831. Journal de la physiologie expérimentale. Ed. Magendie et Méquignon-Marvis, Paris. 11 vol. MU Ca 861 et PR 4129.

MAGENDIE F., 1833. Précis élémentaire de physiologie. 3e Ed. Ed. Méquignon-Marvis, Paris. 1. 1-456. 2, 1-628. FG.

MAGENDIE F., 1839. Du sang et des altérations de ce liquide dans les maladies graves. Leçons professées au Collège de France. Ed. Crochard, Paris. 4, 1-400. FG.

OXFORD ENGLISH DICTIONNARY. 1964. 12 vol. in Fo. Oxford Univ. Press Ed., 1971. FG.

PAWLOW J. P., 1921. Sur le centre de la faim. J. Psych. Norm. Pathol., 18, 273-281.

PLINE I'ANCIEN (23 à 79). Histoire naturelle de Pline. Trad. M. Ajasson de Grandsagne. Ed. Panckoucke, Paris. 1824. INA-PG.

PROUT W., 1824. Sur la nature de l'acide et des bases salifiables qui existent ordinairement dans l'estomac des animaux. Trad. des «Transactions philosophiques pour 1824 ». J. Physiol. Exp. Patho. de F. Magendie. Ed. l'auteur et Méquignon-Marvis, Paris. Tome 4 : 294-299. MU PR 4129.

QUILLET A., 1968. Dictionnaire encyclopédique Quillet. Ed. Lib. A. Quillet. INA-PG.

RICHERAND A., 1801 à 1833. Nouveaux éléments de physiologie. 10 éd. successives. MU $8^{\circ}$ $\mathrm{Bn} 386$.

ROBERT P., 1965. Dictionnaire alphabétique et analogique de la langue française. Ed. Société du nouveau Littré. INA-PG. 8.

RUCKEBUSCH Y., 1963. Recherches sur la régulation centrale du comportement alimentaire chez les Ruminants. Th. Doct. es Sci., Lyon.

RUCKEBUSCH Y., 1979. Historical profile of early digestive studies. Proc. 5th Int. Symp. on Ruminant physiology. MTP Press Ltd., Lancaster. 1-32. FG.

SCHIFF M., 1867. Leçons sur la physiologie de la digestion faites au Muséum d'Histoire Naturelle de Florence. Rédigées par le Dr. Emile Levier. Ed. Hermann Loescher, Florence, Turin. 2 tomes in $8^{\circ}$. Tome $1: 144 \mathrm{pp}$. Tome 2:557 pp. FMD.

SERRES E. R. A., 1832. Recherches d'anatomie transcendante et pathologique. Mém. Acad. Roy. Sci., 11, 585-895 et édition publique 1835. FG.

SOURY J., 1899. Le système nerveux central. Structure et fonctions. Histoire critique des théories et des doctrines. Ed. Carré et Naud, Paris. 1. 1865. FG.

TIEDEMANN F., GMELIN L., 1826. Recherches expérimentales, physiologiques et chimiques sur la

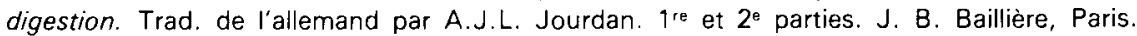
1826-1827. 2 vol. in $8^{\circ}$. Tome 1: 471 pp. + XXIV. MU 4381 et FG.

TRÉSOR DE LA LANGUE FRANÇAISE, 1974. Ed. CNRS. MU.

VULPIAN A., 1866 . Leçons sur la physiologie générale et comparée du système nerveux faites au Muséum d'Histoire Naturelle. Ed. Germer-Baillière, Paris. 1-920. FG.

WILLIEN J. L., 1838. De la faim considérée sous le rapport physiologique, pathologique et thérapeutique. in 4e. Thèse Fac. Méd. Strasbourg. n 15. 48 pp. FMD 90986. 